() Open Access Full Text Article

\title{
Recognizing Prognostic and Predictive Biomarkers in the Treatment of Non-Small Cell Lung Cancer (NSCLC) with Immune Checkpoint Inhibitors (ICls)
}

This article was published in the following Dove Press journal:

Lung Cancer: Targets and Therapy

\author{
Nicholas Giustini \\ Lyudmila Bazhenova \\ UCSD Moores Cancer Center, \\ Department of Hematology and Oncology, \\ 3855 Health Sciences Drive MC \#0987, La \\ Jolla, CA, 92093-0829, USA
}

\begin{abstract}
Immunotherapy plays a central role in the treatment of NSCLC and biomarkers predicting response to ICIs are valuable therapeutic tools. Programmed death-ligand 1 (PDL1) immunohistochemistry (IHC) is integral in therapy selection as its positive predictive nature to ICIs in the metastatic setting is well documented. Tumor mutational burden (TMB) has undergone much study and, while results are somewhat mixed, there is evidence for its positive predictive value with ICI use. Additional markers such as tumor-infiltrating lymphocytes (TILs), gene expression profiling (GEP), mismatch repair (MMR) and microsatellite instability (MSI), somatic mutations, neutrophil to leukocyte ratio (NLR), smoking history, medication history, and immune-related adverse event (irAE) development can further guide clinicians.
\end{abstract}

Keywords: NSCLC, immunotherapy, biomarkers

\section{Introduction}

Lung cancers are the leading cause of cancer-related mortality and, outside of nonmelanoma skin cancer, are the highest incident of all cancers, with NSCLC comprising the majority of all lung cancer cases. ${ }^{1}$ While the advent and utilization of ICIs - antibodies blocking programmed cell death protein 1 (PD-1), PD-L1, and/ or cytotoxic T-lymphocyte-associated protein 4 (CTLA-4) leading to immune cell activation-has significantly improved outcomes, room still exists for improvement. Multiple regimens for front-line treatment of oncogenic driver negative NSCLC are available ranging from mono ICI to dual ICI with platinum doublet chemotherapy in addition to salvage ICI therapy if not used front-line, and choosing the optimal regimen to maximize therapeutic outcomes but minimize side effects can be challenging. ${ }^{2-5}$ While tumoral PD-L1 IHC is one of the most recognized and validated predictive biomarkers in NSCLC, many other pathologic and clinical characteristics are being explored to augment clinician decision-making capabilities for immunotherapeutic regimens.

\section{PD-LI}

PD-L1 testing is done through IHC with different assays utilized with various PD-1 or PD-L1 inhibitors; the mechanism of action of these inhibitors in relation to PD-L1 is
Correspondence: Nicholas Giustini Jolla, CA, 92093-0829

Email ngiustini@health.ucsd.edu
Lung Cancer: Targets and Therapy 2021:12 2|-34 
well described. ${ }^{6}$ The $22 \mathrm{C} 3$ assay pairs with pembrolizumab, 28-8 assay with nivolumab, SP142 with atezolizumab, SP263 with durvalumab, and 73-10 with avelumab. These assays stain for PD-L1 of tumor cells and infiltrating immune cells, with the majority of clinical trials utilizing membranous tumor cell staining for stratification, except for atezolizumab and the SP142 assay. Blueprint Phase I and II trials were conducted to evaluate the equivalence of these assays noting that the 22C3, 28-8, and SP263 assays are fairly well correlated. The SP142 assay was noted to have less sensitivity for PD-L1 staining and as a result underestimates PD-L1 compared to the $22 \mathrm{C} 3,28-8$, and SP263 assays. The 73-10 assay was noted to have a higher sensitivity for PD-L1 staining and as a result overestimates PD-L1 compared to the $22 \mathrm{C} 3,28-8$, and SP263 assays. ${ }^{7,8}$

For stage III unresectable NSCLC, chemoradiation followed by a year of durvalumab monotherapy or placebo produced impressive improvements in median progression-free survival (PFS) and overall survival (OS). However, in a post hoc analysis dividing PD-L1 $\geq 1 \%$ versus $<1 \%$, the PFS hazard ratio (HR) was $0.46(95 \%$ CI $0.33-0.64$ ) for $\geq 1 \%$ vs 0.73 (95\% CI $0.48-1.11$ ) for $<1 \%$; the OS HR was 0.59 (95\% CI $0.41-0.83$ ) for $\geq 1 \%$ vs 1.14 (95\% CI $0.71-1.84)$ for $<1 \%$. While these data utilize subgroup analysis in a post hoc setting, they do highlight the predictive value of PD-L1 IHC for ICI therapy. ${ }^{9}$

Multiple studies have shown the benefit of ICI therapy in 2nd line or later setting, including with pembrolizumab, nivolumab, atezolizumab, and avelumab. In the Phase III JAVELIN Lung 200 trial of avelumab versus docetaxel in NSCLC previously treated with platinum chemotherapy, there was an overall response rate (ORR) improvement with avelumab, however no benefit in the PD-L1 $\geq 1 \%$ cohort for PFS or OS. Upon subgroup analysis of PD-L1 $\geq 50 \%$ and $\geq 80 \%$ cohorts, the odds ratio for ORR increased and both median PFS and OS were improved in the avelumab arm. For instance, for OS, the HR for PD-L1 $\geq 1 \%$ was 0.90 (95\% CI $0.72-1.12$ ), for PD-L1 $\geq 50 \%$ was 0.67 (95\% CI 0.51-0.89), and for PD-L1 $\geq 80 \%$ was 0.59 (95\% CI 0.42-0.83). ${ }^{10}$ Similar trends with increasing PD-L1 were observed in the earlier 2 nd line studies of nivolumab in CheckMate 057, pembrolizumab in Keynote 010, and atezolizumab in OAK; however, the predictive value of PD-L1 for ICI therapy was not demonstrated in the squamous NSCLC population treated with nivolumab in CheckMate $017 .{ }^{11-14}$ A meta-analysis of various PD-1 and PD-L1 agents versus docetaxel used 2nd line or later with PD-L1 IHC stratification noted that either ICI led to an OS advantage; however, a PFS advantage was only seen with PD-1 but not PD-L1 inhibitors. ${ }^{6}$

PD-L1 IHC as a predictive biomarker for front-line ICI therapy was demonstrated with the combination of pembrolizumab with platinum chemotherapy in two phase III Keynote studies. The nonsquamous Keynote 189 evaluated differences in ORR, PFS, and OS in the PD-L1 $<1 \%$, $1-49 \%$, and $\geq 50 \%$ cohorts. For both ORR and median PFS, increasing PD-L1 was associated with stepwise improvements in the pembrolizumab arm. With median OS, there were similar improvements across the board for PD-L1 in the pembrolizumab cohort; however, this may have been confounded by the $53.9 \%$ control postprogression crossover. ${ }^{3}$ The squamous Keynote 407 noted that while ORR was similar across PD-L1 subgroups, median PFS and OS showed improvements in HR in the pembrolizumab treated groups of PD-L1 $\geq 1 \%$ vs PD-L1 $<1 \%{ }^{15}$

The phase III Keynote 042 study of pembrolizumab monotherapy versus platinum chemotherapy in PD-L1 $\geq 1 \%$ NSCLC also demonstrated the predictive quality of PD-L1 IHC. For median PFS, the PD-L1 $\geq 1 \%$ group was not improved compared to chemotherapy; however, in the $\geq 50 \%$ subgroup, the HR was statistically improved. For median OS, the HR of the PD-L1 $1-49 \%$ group was nonsignificant at 0.92 (95\% CI 0.77-1.11), while the HR of the PD-L1 $\geq 50 \%$ was significant at 0.69 (95\% CI $0.56-0.85) .{ }^{16}$ Keynote 024 , a study of upfront pembrolizumab monotherapy versus chemotherapy in NSCLC with PD-L1 $\geq 50 \%$ confirmed a median OS HR of $0.63(95 \%$ CI $0.47-0.86)^{2}$

While no prospective trials have been powered to compare outcomes for NSCLC patients with PD-L1 IHC gradations above $50 \%$, a multicenter retrospective analysis of pembrolizumab in this patient group was conducted. In the cohort of 187 patients, the median PD-L1 was $80 \%$; responders median PD-L1 was $90 \%$ compared to $75 \%$ in nonresponders. When looking at quartiles of PD-L1 75-100\% versus $50-74 \%$, the ORR was $53.6 \%$ vs $30.7 \%$, median PFS HR was improved at 0.63 (95\% CI 0.43-0.92) and median OS trended towards but did not reach significance with an HR of 0.62 (95\% CI 0.37-1.03). Furthermore, when looking at PD-L1 90-100\% versus 50-89\%, the ORR was $60 \%$ vs $32.7 \%$, median PFS was improved with an HR of 0.50 (95\% CI 0.33-0.74), and median OS was improved with an HR of 0.39 (95\% CI 0.21-0.70). ${ }^{17}$ These data complete the picture that increasing PD-L1 predicts improved outcomes with ICI therapy, though even in the PD-L1 
90-100\% group, $40 \%$ of patients did not experience a response, indicating PD-L1 as an incomplete immunotherapeutic biomarker. Further prospective studies to confirm these findings across diverse populations would deepen understanding within the field. ${ }^{18}$

\section{TMB}

TMB evaluates the nonsynonymous mutation burden of a particular neoplasm via either tissue TMB (tTMB) or blood TMB (bTMB) analysis and is reported as mutations (mut) per megabase (mb) of DNA or per whole exome. As TMB increases, tumor neoantigen load increases, which the immune system can theoretically recognize and target especially under encouragement from ICIs. One of the early studies of tTMB noted that the tTMB of NSCLC patients was higher in those with either a response or stable disease to ICIs compared to those who progressed. If these patients were then separated into tTMB above and below the median, ORR and median PFS were statistically improved. $^{19}$

A retrospective analysis of tTMB in multiple solid tumors treated with single or dual agent ICIs noted almost across the board improvements in OS. Rather than using median tTMB, this study evaluated upper percentiles of tTMB compared to lower percentiles per tumor type. For instance, in NSCLC, when evaluating the top $10^{\text {th }}$ percentile of tTMB - correlating to a tTMB of $20.2 \mathrm{mut} / \mathrm{mb}$ or higher-compared to the bottom $90^{\text {th }}$ percentile tTMB group, OS was significantly improved. This difference was also present, though not as pronounced, in the top $30^{\text {th }}$ percentile - tTMB of $10.2 \mathrm{mut} / \mathrm{mb}$ or higher. When evaluated as a continuous variable, tTMB in NSCLC treated with ICI therapy was associated with an improvement in $\mathrm{OS}^{20}$

However, contradictions in available data make the use of TMB as a stand-alone predictive biomarker premature. The prospective phase III trial CheckMate 227 evaluated the use of different combinations of ipilimumab, nivolumab, and platinum chemotherapy in the frontline treatment of NSCLC with a coprimary PFS endpoint of ipilimumab plus nivolumab versus chemotherapy evaluated in tTMB $\geq 10$ or $<10 \mathrm{mut} / \mathrm{mb}$ groups. Initial reports noted an improvement in PFS in the ICI group compared to chemotherapy only in the tTMB $\geq 10 \mathrm{mut} / \mathrm{mb}$ group; however, this did not translate to a differential improvement in OS between the groups. ${ }^{4,21}$

Indeed, the role of tTMB as a predictor of response to pembrolizumab in NSCLC is mixed. A retrospective analysis of a pooled cohort of NSCLC patients treated with either pembrolizumab plus chemotherapy versus chemotherapy in Keynotes 021, 189, and 407 showed tTMB did not predict ORR, PFS, or OS when evaluated as either a continuous variable or when divided into subgroups of $\geq 175$ mut/exome vs $<175$ mut/exome. ${ }^{22}$ Alternatively, when retrospectively evaluating a pooled cohort of NSCLC patients with PD-L1 $\geq 1 \%$ treated with pembrolizumab versus chemotherapy in Keynotes 010 and 042 , tTMB predicted both PFS and OS both when tTMB was considered as a continuous variable and when divided into subgroups of $\geq 175$ mut/exome versus $<175$ mut/exome. ${ }^{23}$

Separately, an evaluation of bTMB as a biomarker for atezolizumab was undertaken as an alternative to tTMB by retrospectively assessing the POPLAR and OAK studiesboth evaluated atezolizumab versus docetaxel after at least first-line therapy in NSCLC - using a cut point of bTMB $\geq 16 \mathrm{mut} / \mathrm{mb}$. In the Phase II POPLAR study, though bTMB trended towards significance for PFS, it was not a predictor of OS. In the phase III OAK study, bTMB was a predictor of PFS benefit in the ICI group but was not significant for OS. ${ }^{24}$ An analysis of front-line durvalumab plus tremelimumab versus chemotherapy in the phase III MYSTIC trial in NSCLC noted no predictive value of ICI effect on PFS or OS with a tTMB $\geq 10 \mathrm{mut} / \mathrm{mb}$. However, ORR, PFS, and OS were improved in the bTMB high group when using a bTMB cutoff of $\geq 20 \mathrm{mut} / \mathrm{mb}^{25}$

Taken together, these data suggest for ICI use in NSCLC, TMB is a positive predictive biomarker for PFS. The data are mixed with regards to OS as an outcome; however, there is an indication that higher TMB cutoffs of the top $10^{\text {th }}$ percentile or $\mathrm{TMB} \geq 20 \mathrm{mut} / \mathrm{mb}$ are more realistic to use as predictors of OS. However, more prospective studies are needed to validate these results.

\section{TILs and GEP}

TILs have been demonstrated to be a prognostic biomarker before the ICI era with stromal infiltration of CD8+ and CD4+ lymphocytes associated with improved disease-specific survival in early-stage NSCLC. ${ }^{26}$ A separate analysis of resected NSCLC treated with adjuvant chemotherapy noted with intense tumor lymphocytic infiltration both a longer diseasefree survival (DFS) with an HR of 0.55 (95\% CI 0.41-0.73) and OS with an HR of 0.53 (95\% CI 0.39-0.73) compared to nonintense infiltration. ${ }^{27} \mathrm{~A}$ meta-analysis of 24 studies found elevated tumoral $\mathrm{CD} 3+, \mathrm{CD} 4+$, and CD8+ lymphocytes were associated with an improvement in OS while increased FoxP3 + regulatory lymphocytes were associated with a worsening of 
OS. ${ }^{28}$ Similarly, through evaluating previously validated GEP of lymphocytes and macrophages via mRNA sequencing expression of The Cancer Genome Atlas (TCGA), multiple tumors including NSCLC exhibited survival benefit if tumors scored high in these signatures. ${ }^{29}$

The predictive value of TILs and GEP is less clear than the prognostic value; however, both are predicated on the tumoral invasion of certain lymphocytes leading to improved immune surveillance. One retrospective of NSCLC patients treated with nivolumab noted an improved ORR with an increased CD8+/CD4+ ratio compared to a low ORR with a lower ratio. ${ }^{30}$ Another noted that responders to nivolumab displayed a low lymphocytic PD-1/CD8+ ratio compared to nonresponders. ${ }^{31}$ Evaluation of TILs and GEP is ongoing and further data are needed to elucidate their role as biomarkers.

\section{MMR and MSI}

MMR and MSI status have been thoroughly studied in tumors with the highest prevalence of MMR deficient (dMMR) or MSI high (MSI-H) malignancies, namely colon and other gastrointestinal malignancies, as well as endometrial cancer with the mechanism of response to ICI well described in the literature. By contrast, the evaluation of treatment of dMMR and MSI-H in NSCLC has been limited by low prevalence and thus is rarely considered in most treatment algorithms. ${ }^{32}$ A phase II study of 20 previously treated patients with metastatic disease evaluated the use of pembrolizumab in MSI-H colon patients and MSI-H non-colon patients. While the cohorts were small, colon patients exhibited an ORR of $40 \%$ and a 20 -week PFS of $78 \%$, while non-colon patients demonstrated an ORR of $71 \%$ and a 20 -week PFS of $67 \% .^{33}$ A larger phase II study evaluated 78 previously treated metastatic patients including an MSI-H colon cohort and a more diverse MSI-H non-colon cohort. Outcomes were similar between the cohorts with a combined ORR of $53 \%$, disease control rate (DCR) of $77 \%$, and complete response (CR) rate of $21 \%$. The two-year median PFS was 53\% and the two-year median OS was $64 \% .^{34}$

While neither of these studies included patients with NSCLC, multiple retrospective analyses have attempted to quantify the prevalence of MSI-H in NSCLC, as these tumors would likely benefit from ICI therapy. In a study of 297 EGFR, KRAS, and BRAF wildtype NSCLC patients, $3(1 \%)$ were identified as MSI-H, all previous smokers. ${ }^{35}$ A separate analysis of 480 pulmonary adenocarcinoma patients identified $4(0.83 \%)$ MSI-H cases, again all previous smokers. ${ }^{36}$ Finally, in one of the largest analyses of 1868 NSCLC patients, $12(0.6 \%)$ were MSI-H. ${ }^{32}$

\section{Somatic Mutations}

One of the main predictors of poor response to immunotherapy is the presence of certain oncogenic drivers. In a retrospective analysis of NSCLC patients treated with ICI therapy, the differential response to EGFR mutated and ALK rearranged versus wildtype for both was assessed. The ORR for EGFR or ALK driver patients was 3.6\%, a single unconfirmed response, compared to $23.3 \%$ in the wildtype population when treated with single-agent ICI therapy. ${ }^{37}$ The IMMUNOTARGET retrospective analysis evaluated ICI therapy largely in the 2 nd or 3rd line in metastatic NSCLC patients with oncogenic driver alterations including KRAS, EGFR, BRAF, HER2, MET, ALK, ROS1, and RET. In this study, the ORR was $19 \%$, which decreased to $12.7 \%$ if KRAS was excluded. Evaluating by 12-month PFS, KRAS with 25.6\% and MET with 23.4\% exhibited the best outcomes, HER2 with $18.0 \%$ exhibited an intermediate outcome, and RET with $7.0 \%$, EGFR with $6.4 \%$, ALK with $5.9 \%$, and ROS1 unevaluable due to small numbers exhibited the poorest PFS outcomes. ${ }^{38}$ In a phase II prospective analysis of patients with EGFR mutations and positive PD-L1 IHC treated with first-line pembrolizumab, the ORR was 0/10 and median PFS 119 days with the study closing early due to futility. ${ }^{39}$ Finally, in a meta-analysis of EGFR mutated NSCLC patients included in the second line ICI monotherapy versus docetaxel studies of CheckMate 057, Keynote 010, and POPLAR, the OS HR was 1.05 (95\% CI 0.70-1.55). ${ }^{40}$

NSCLC patients with STK11 (also known as LKB1) and KEAP1 mutations seem to perform worse compared to controls; however, data are mixed whether these are negative predictive or prognostic biomarkers. In an analysis of KRAS mutated NSCLC patients treated with ICI therapy, those with co-mutations in STK11 exhibited a worse ORR of $7.4 \%$ vs $28.6 \%$, shorter PFS with an HR of $1.98(95 \%$ CI 1.33-2.94), and shorter OS with an HR of 1.99 (95\% CI 1.29-3.06) when compared to KRAS mutated STK11 wildtype patients. When evaluating these patients in the CheckMate 057 cohort of nivolumab versus docetaxel, while there was a significant difference in ORR in the nivolumab arm, there was not in the docetaxel arm possibly owing to sample size. KRAS/STK11 co-mutated patients were also noted to have a significantly lower rate of PD-L1 positivity; however, even in a PD-L1 IHC 
positive NSCLC cohort treated with ICI therapy, ORR ( $0 \%$ vs $34.5 \%$ ), PFS (HR 4.76 95\% CI 2.0-11.1), and OS (HR 14.3 95\% CI 3.4-50.0) were worsened with STK11 mutants compared to controls. ${ }^{41}$

A separate analysis of KRAS mutated NSCLC patients identified that patients were commonly co-mutated with either STK11, KEAP1/NFE2L2, or both. On multivariate analysis, OS was only independently shortened for those with KEAP1/NFE2L2 mutations with an HR of $1.96(95 \%$ CI 1.33-2.92) and was similarly shortened in both ICI and chemotherapy groups. ${ }^{42}$ A study of nonsquamous NSCLC patients in a FoundationOne database identified 30\% of patients with either or both STK11 and KEAP1 mutations. These patients more commonly exhibited PD-L1 negative IHC staining. PFS and OS were worse in mutated patients when treated with ICI or chemotherapy, with the worst outcomes found in co-mutated patients. ${ }^{43}$ An analysis of NSCLC patients in the MYSTIC trial of NSCLC patients treated with durvalumab plus tremelimumab, durvalumab, or platinum doublet chemotherapy evaluated subgroups of STK11 or KEAP1 mutated patients. The OS of KEAP1 and STK11 mutated patients were shorter than controls with HRs of 1.64 (95\% CI 1.37-1.97) and 1.52 (95\% CI 1.25-1.85) respectively. Upon subgroup analysis, there was no significant difference in outcomes between single or dual ICI therapy versus chemotherapy. ${ }^{44}$

Retrospective data suggest ARID1A alterations appear to both compromise MMR proteins and lead to increased TMB. ${ }^{45}$ A separate analysis of ARID1A and ARID1B noted that while these mutations in NSCLC were previously negatively prognostic, they may now be positive predictive for ICIs. On treatment with ICI therapy, ARID1A mutated NSCLC patients exhibited improved ORR and similar PFS to controls, while ARID1B mutated NSCLC patients experienced both improved ORR and PFS. $^{46}$ In the MYSTIC trial subgroup analysis, while there was no significant OS difference in ARID1A mutated patients versus controls, treatment of ARID1A mutated NSCLC patients with dual ICIs compared to chemotherapy portended an OS benefit with an HR of $0.42(95 \%$ CI $0.24-0.76){ }^{44}$

Mutations that affect the DNA damage response (DDR) system-eight enzymatic pathways related to DNA upkeep - lead to increased neoantigen load, TMB, and immune gene expression signatures. In an analysis of 34 NSCLC patients treated with ICI therapy, those with two mutations in DDR - base excision repair and mismatch repair-exhibited an ORR of $63.6 \%$ compared to
$21.7 \%$ and median PFS HR of 0.21 (95\% CI $0.06-0.71)$ compared to controls. ${ }^{47}$

In NSCLC tumors, those with ZFHX3 mutations exhibited significantly increased numbers of DDR pathway mutations and increased TMB compared to ZFHX3 wildtype controls. In a cohort of 350 NSCLC patients treated with single or dual ICIs, median OS was significantly longer in ZFHX3 mutants compared to wildtype patients with an HR of 0.26 (95\% CI 0.17-0.41). In TCGA cohort, a non-ICI treated NSCLC cohort, ZFHX3 mutant status was not associated with improved OS, indicating a possible predictive biomarker for ICI therapy. ${ }^{48}$

In a separate analysis of NOTCH1/2/3/4 mutations in NSCLC, inactivating NOTCH $1 / 2 / 3$ mutations were associated with increased TMB as well as increased activation of the DDR system and subsequent mutagenesis. Clinically, in a pooled analysis of multiple NSCLC cohorts treated with ICI therapy, outcomes were improved with inactivating NOTCH1/2/3 mutations with increased ORR with a RR of 2.2 (95\% CI 1.39-3.51), longer PFS with an HR of 0.61 (95\% CI $0.46-0.81$ ), and longer OS with an HR of 0.56 (95\% CI $0.32-0.96$ ). In a multivariate analysis of inactivating NOTCH1/2/3 mutations in the POPLAR and OAK NSCLC studies evaluating atezolizumab versus docetaxel, NOTCH1/2/3 mutant patients were independently associated with improved PFS and OS in the atezolizumab group but not the docetaxel group. ${ }^{49}$

\section{NLR}

NLR is thought to be a prognostic and potentially predictive biomarker with regards to immunotherapy in NSCLC. Theoretically, lymphopenia reflects impaired cell-mediated immunity, while neutrophilia reflects systemic inflammation with decreased tumoral lymphocytic infiltration, thus one would expect worsened outcomes with an increased NLR. A meta-analysis of NSCLC patients prior to the immunotherapy era noted that pretreatment NLR predicted a shorter PFS with an HR of 1.63 (95\% CI 1.27-2.09) and OS with an HR of 1.70 (95\% CI 1.39-2.09) with the cut off of NLR $>5$ reducing heterogeneity. ${ }^{50}$

Similarly, a recent meta-analysis of largely retrospective studies of ICI use in NSCLC noted both worsened PFS and OS with elevated NLR. The median and cut-off NLR of most studies assessed was 5.0. Median PFS was 1.9 months vs 4.7 months with an HR of 1.59 (95\% CI 1.36-1.82). Median OS was 4.7 months versus 16.0 months with an HR of 2.07 (95\% CI 1.70-2.44). Both pretreatment and posttreatment NLR resulted in similar 
HRs for PFS and OS. ${ }^{51}$ In a separate retrospective evaluating outcomes in NSCLC patients treated with pembrolizumab with PD-L1 IHC $\geq 50 \%$, both PFS and OS were statistically worsened on multivariate analysis in the NLR high group with HRs of 2.214 (95\% CI 1.04-4.74) and 3.225 (95\% CI 1.34-7.76) respectively. ${ }^{52}$ These data indicate NLR is a prognostic biomarker with further study needed to elucidate if NLR could be used as a predictive immunotherapy biomarker in NSCLC.

\section{Smoking History}

While many clinical characteristics - such as sex, race, and age-have been evaluated as possible predictors of response to immunotherapy, perhaps the only characteristic with significant evidence as a predictive biomarker to ICI therapy is smoking history. A meta-analysis of four phase III trials of 2 nd line single ICI vs chemotherapy in NSCLC showed improvements in PFS and OS. In smokers, the PFS HR was 0.55 (95\% CI 0.43-0.67) and OS HR was 0.69 (95\% CI 0.60-0.78). In nonsmokers, the PFS HR was 0.90 (95\% CI 0.11-7.59) and OS HR was 0.80 (95\% CI $0.54-1.06) .{ }^{53}$ A separate phase II study of NSCLC patients with 2nd line nivolumab noted higher ORR, PFS, and OS in smokers compared to nonsmokers. ${ }^{54}$ Another meta-analysis of six mixed $1 \mathrm{st}$ and 2nd line single ICI vs chemotherapy trials in NSCLC also showed an OS benefit in the smoking group with an HR of 0.70 (95\% CI 0.63-0.89) but not the nonsmoking group with an HR of 0.79 (95\% CI 0.59-1.09). For the PFS analysis only two 1st line studies were included, which showed no benefit with a PFS HR of $0.85(95 \% 0.71-1.01)$ in smokers but a detriment to PFS in nonsmokers with an HR of $2.30(95 \%$ CI $1.23-4.28)^{55}$

In 2020, a meta-analysis of largely phase III randomized control trials in smokers versus nonsmokers in solid tumors was published. Of the 17 trials, 13 trials were of NSCLC. The OS data for ICI monotherapy compared to chemotherapy showed a benefit in smokers with an OS HR of 0.79 (95\% CI 0.69-0.89) while there was no benefit for nonsmokers with an OS HR of 1.01 (95\% CI 0.84-1.21). For OS of chemoimmunotherapy compared to chemotherapy, both smokers and nonsmokers received a benefit with HRs of 0.72 (95\% CI 0.61-0.85) and 0.45 (95\% CI 0.28-0.71) respectively. ${ }^{56}$

Caveats to these analyses include relatively small sample sizes for the nonsmoking population and variable testing and inclusion of patients with oncogenic drivers, such as EGFR and ALK. The IMMUNOTARGET retrospective analysis identified an improvement of PFS in HER2 or BRAF smokers compared to nonsmokers treated with immunotherapy and no change in PFS with other common alterations and smoking, though these were subgroup analyses. $^{38}$

A retrospective analysis of NSCLC patients treated with ICI therapy noted on multivariate analysis that a positive smoking history demonstrated a positive effect on ORR. Additionally, TMB $\geq 10$ mut/mb was significantly associated with a longer smoking history. ${ }^{57}$ A separate retrospective analysis of NSCLC patients noted when testing TMB as a continuous variable, smoking history was the only associated significant factor. ${ }^{58}$ In addition to the association with TMB, a review of multiple studies concluded there is also a positive association between smoking history and PD-L1 IHC. ${ }^{59}$

When assessing a previously validated smoking-related molecular signature for improvements in outcomes, a small retrospective analysis showed these smokingrelated signatures correlated to improvements in ORR and PFS; however, this was not borne out in patientrelated identification of smoking history. ${ }^{19}$ There is likely a link between smoking-related NSCLC and both increased TMB and PD-L1 IHC with concomitant decreased rates of actionable driver mutations. These factors enrich for a group of patients who experience improved outcomes with ICIs; however, it remains unclear if there are additional unknown factors at play with smoking-related NSCLC that predict improved outcomes.

\section{Antibiotics and the Microbiome}

While incompletely understood, evidence suggests that the interplay between the microbiome and the immune system can affect the efficacy of ICIs in multiple malignancies, including NSCLC. To further explore this interaction, multiple studies have evaluated how medications that can alter the microbiome-namely antibiotics and proton pump inhibitors - can affect clinical outcomes.

Numerous retrospective analyses have evaluated antibiotic exposure effects on immunotherapy response and survival with varying definitions of exposure, with most studies investigating antibiotics used variably 1-3 months prior to and after the first infusion of an ICI. A metaanalysis of solid oncology patients treated with ICIs indicated that prior or intercurrent antibiotic therapy was significantly associated with a worsened median PFS with an 
HR of 1.53 (95\% CI 1.22-1.93) and a worsened median OS with an HR of 2.07 (95\% CI 1.51-2.84). ${ }^{60}$

There are notably three studies indicating no difference in ICI outcome with antibiotic exposure. One study of 74 NSCLC patients treated with nivolumab evaluated antibiotic use starting 3 months prior to ICI infusion until progression and noted no difference in ORR, median PFS, or median OS. ${ }^{61}$ A separate evaluation of 90 NSCLC patients treated with nivolumab monitoring antibiotics 1 month prior to ICI infusion noted no difference in median PFS or OS. ${ }^{62}$ Of studies evaluating antibiotic use 1 month before or after ICI infusion in NSCLC patients, one study of 142 patients noted no difference in median PFS or OS, while two other studies of 30 and 109 patients, respectively, noted worsening in the median in PFS and OS with antibiotic exposure. ${ }^{63-65}$

A separate study of ICI in both RCC and predominantly NSCLC evaluated antibiotics used at time points both 30 days and 60 days prior to the first ICI infusion. Antibiotic use within the 30 days prior to ICI therapy was associated with a significantly worsened median OS but not PFS, while antibiotic use 60 days prior was associated with an improving but still statistically worsened OS. ${ }^{66}$ These data suggest that dysbiosis associated with antibiotic use may be reversible given enough time for the microbiome to recapitulate.

Interestingly, a study of largely NSCLC treated with ICIs monitored antibiotic use 1 month prior to the first ICI infusion and then intercurrently noted worsened ORR and median OS only with antibiotics prior to ICI therapy; with intercurrent antibiotic use, no survival difference was seen. ${ }^{67}$ A separate study of ICIs in NSCLC evaluated the use of antibiotics 1 month prior to 3 months post first ICI infusion. While ORR, median PFS, and median OS were numerically worse in the month prior to ICI, these data were not statistically significant. However, an analysis of the ratio of days receiving antibiotics to days receiving ICI noted that an increasing ratio was associated with a statistically worsened median PFS and OS; these data were confirmed on multivariate analysis when setting the cut-off evaluation as the median ratio. ${ }^{68} \mathrm{~A}$ third study evaluating ICI in melanoma, NSCLC, and RCC patients studied the effect of antibiotics given 2 weeks before to 6 weeks after the first ICI infusion. This study noted that median PFS and OS were worsened with antibiotic use. Subgroups were evaluated by separating patients into no antibiotic use versus one antibiotic use for less than 7 days versus multiple antibiotics, antibiotics lasting seven or more days, or multiple courses of antibiotics. A single antibiotic for less than 7 days noted numerical but not statistical worsening compared to no antibiotic use; however, multiple antibiotics, multiple courses of antibiotics, or a long course of antibiotics were associated with worsened outcomes. Taken together these data indicate a cumulative effect on the microbiome that is more pronounced with antibiotics prior to ICI, the use of multiple antibiotics, or long courses of antibiotics that leads to poorer outcomes with ICI therapy. ${ }^{69}$ There has also been a less rigorous evaluation of the effect of PPI use on ICI efficacy in NSCLC given its effect on the microbiome; however, the data are mixed and indicate no convincing effect on clinical outcomes. ${ }^{65,70,71}$ Table 1 summarizes the effect of antibiotics on ICI efficacy.

Multiple studies have evaluated how changes in the microbiome can affect responses to ICIs in NSCLC. A microbiota analysis of 60 NSCLC and 40 RCC patients receiving ICIs investigated responders versus nonresponders. Responders demonstrated an overrepresentation of certain bacteria including Firmicutes, Alistipes, and Akkermansia with Akkermansia muciniphila most associated with favorable outcomes. Evaluating by the best response, A. muciniphila was detected in the stool of $69 \%$ of patients with partial response, $58 \%$ of patients with stable disease, and $34 \%$ of patients with progressive disease, and was also significantly associated with a longer PFS. Stool from responders and nonresponders was transplanted into germ-free mice inoculated with tumor cells and then treated with ICI therapy with resultant delay in tumor growth only in mice with stool from responders. ${ }^{71}$ Similarly, a separate analysis studied the stool of responders versus nonresponders to ICIs in NSCLC and noted increased diversity of the microbiota was associated with increased response and improved median PFS. ${ }^{72}$ A study evaluating antibiotic use and plasma citrulline levels in NSCLC patients receiving nivolumab noted that peritreatment antibiotics correlated with decreased plasma citrulline levels, which were associated with a worse OS. It was posited that citrulline may act as a marker of enterocyte function and low levels may correspond with dysbiosis. ${ }^{73}$ Finally, a study evaluating the antitumor effect of Enterococcus gallinarum (MRx0518) on mouse models of lung, renal, and breast cancer noted that mice exposed to this bacterium reduced tumor size $35-51 \%$ compared to controls and this response was associated with an increased tumor $\mathrm{CD} 8+\mathrm{T}$ cell:Treg ratio. ${ }^{74}$ 


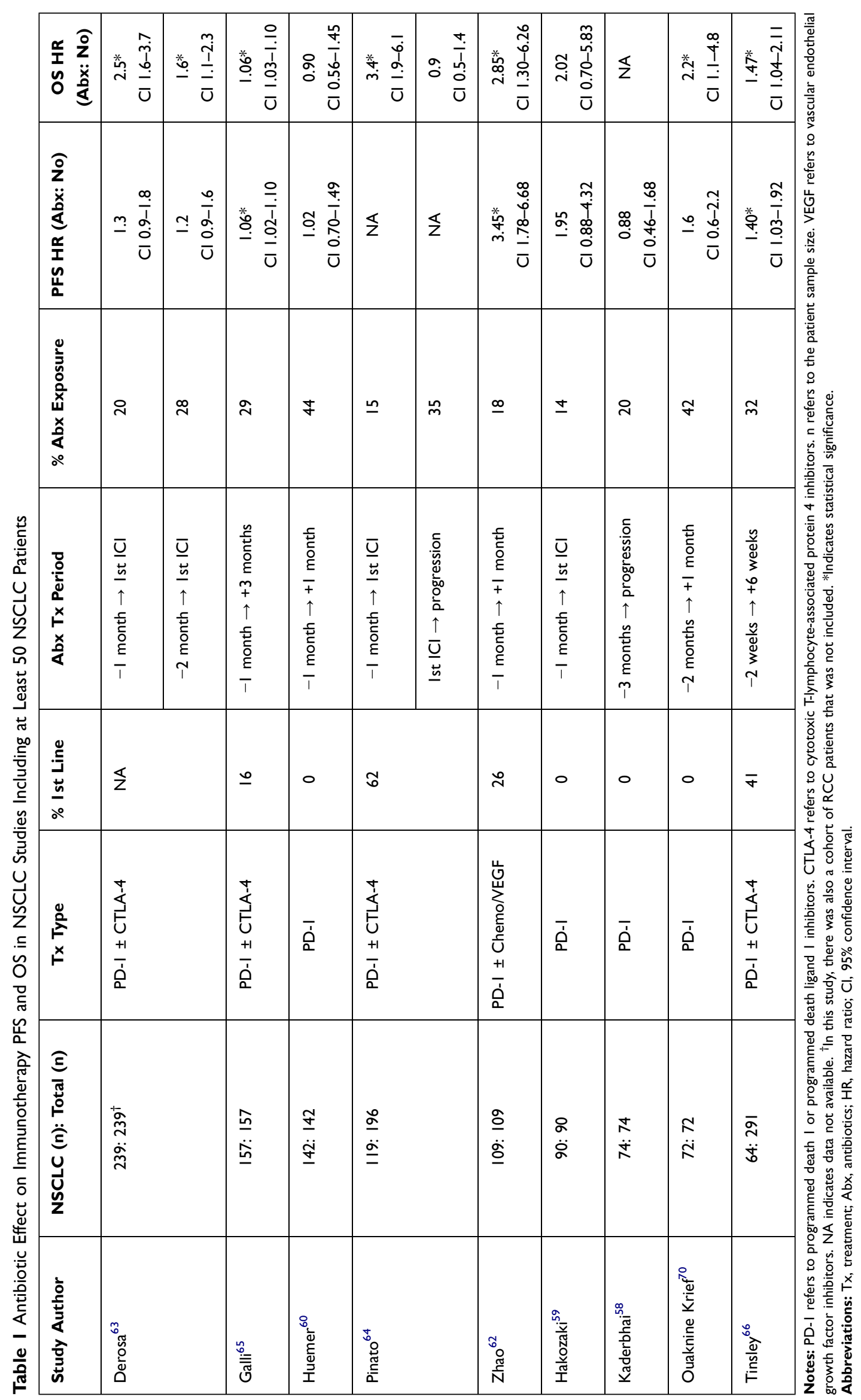




\section{Corticosteroids and irAEs}

Given the immunosuppressive effect of corticosteroids which is taken advantage of when treating irAEs, many studies evaluating the efficacy of ICIs excluded patients receiving supraphysiologic doses of these drugs. Multiple studies have evaluated if baseline corticosteroid use or intercurrent corticosteroids with ICIs affect clinical outcomes, as well as if the development of irAEs portends a difference in clinical outcomes given the evident excitation of the immune system. With regard to corticosteroids, most studies set the threshold for exclusion at greater than or equal to a prednisone equivalent of $10 \mathrm{mg}$ daily.

A meta-analysis of largely NSCLC and melanoma studies noted any corticosteroid use during ICI therapy was associated with a worsened median PFS and OS with HRs of 1.34 (95\% CI 1.02-1.76) and 1.54 (95\% CI 1.24-1.91) respectively, with corticosteroid use for irAE mitigation suggesting no difference in OS with an HR of 1.08 (95\% CI $0.79-1.49) .{ }^{75}$ Three retrospective analyses with limited data regarding the dose of prednisone administered to NSCLC patients treated with ICI monotherapy nevertheless noted those receiving corticosteroids on the day of ICI infusion exhibited a worsened median PFS. In two of these studies, median OS was also significantly worsened. One subgroup analysis confirmed the worsening of median PFS and OS maintained significance in a cohort of NSCLC patients with brain metastases. ${ }^{76-78}$

One study evaluated the impact of baseline prednisone within the 30 days prior to the first ICI infusion in NSCLC patients. In the cohort from MSKCC, there was a worsened ORR, median PFS, and median OS in patients receiving at least prednisone $10 \mathrm{mg}$ daily and in the GRCC cohort there was a worsened median PFS and OS. In the pooled cohort, patients receiving prednisone $\geq 20 \mathrm{mg}$ daily compared to 10-19 mg daily did equally poorly. If patients received corticosteroids in the 30 days prior to ICI infusion but stopped them prior to the day of infusion, patients had intermediate outcomes between controls and patients who continued corticosteroids on the day of ICI infusion. ${ }^{79}$

A study of NSCLC patients receiving corticosteroids during the first 28 days of ICI therapy found those receiving the equivalent of $10 \mathrm{mg}$ of prednisone or more daily experienced a worsened DCR, median PFS, and median OS. ${ }^{80}$ A similar study noted that ICI-treated NSCLC patients receiving greater than the equivalent of prednisone $10 \mathrm{mg}$ daily during the first 30 days of ICI therapy received fewer doses of immunotherapy and exhibited a worse median OS.
However, for patients who started corticosteroids for an irAE in the first 30 days of ICI therapy, there was no difference with regards to outcomes compared to controls. ${ }^{81}$

Another study noted similar findings of poorer outcomes with corticosteroid use prior to ICI therapy and, while patients receiving intercurrent steroids with ICI therapy had worse outcomes, this was driven by patients receiving steroids for cancer-related symptoms (brain metastases and cancer-related dyspnea, pain, and fatigue) and not for those receiving steroids for irAEs or COPD flares. ${ }^{82}$ These findings of worsened outcomes with the use of corticosteroids for cancer-related symptoms but not for cancer unrelated symptoms were corroborated in a separate retrospective analysis. ${ }^{83}$ A third study confirmed that systemic corticosteroids for patients receiving ICI were only associated with worsened outcomes for non irAE indications. ${ }^{84}$ Taken together, these data suggest the use of prednisone $10 \mathrm{mg}$ daily or greater prior to receiving ICI is associated with poorer outcomes; however, starting corticosteroids while already on ICI therapy has no effect on the outcome if given for an irAE. If intercurrent corticosteroids were given while on ICI therapy for cancer-related symptoms such as brain metastases, outcomes were worse; however, this may be an incidental correlation unrelated to the corticosteroid inhibition of the immune response on the malignancy. Table 2 summarizes the effect of corticosteroids on ICI efficacy.

In fact, the development of an irAE while receiving ICI therapy for NSCLC may be positively predictive. A retrospective study noted that patients who developed an irAE within 6 weeks of starting ICIs experienced significantly improved ORR, median PFS, and median OS. ${ }^{85}$ A separate analysis noted that for patients who developed an irAE during ICI treatment, ORR, DCR, median PFS, and median OS were all improved. There was also a nonsignificant trend towards longer OS if the onset of the irAE was more than 3 months after starting ICI therapy. ${ }^{86}$ Another study again noted improvement in ORR, DCR, median PFS, and median OS in patients who developed an irAE on ICIs. Furthermore, those patients who developed at least two irAEs had improved outcomes compared to patients with a single irAE, and those with a single irAE exhibited better outcomes than patients who developed no irAE. ${ }^{87}$ These studies performed subgroup analyses on the type of irAE that exhibited better outcomes and generally cutaneous and endocrinological irAEs performed better.

Finally, a recent study redemonstrated improvement in ORR, DCR, duration of response, median PFS, and median 


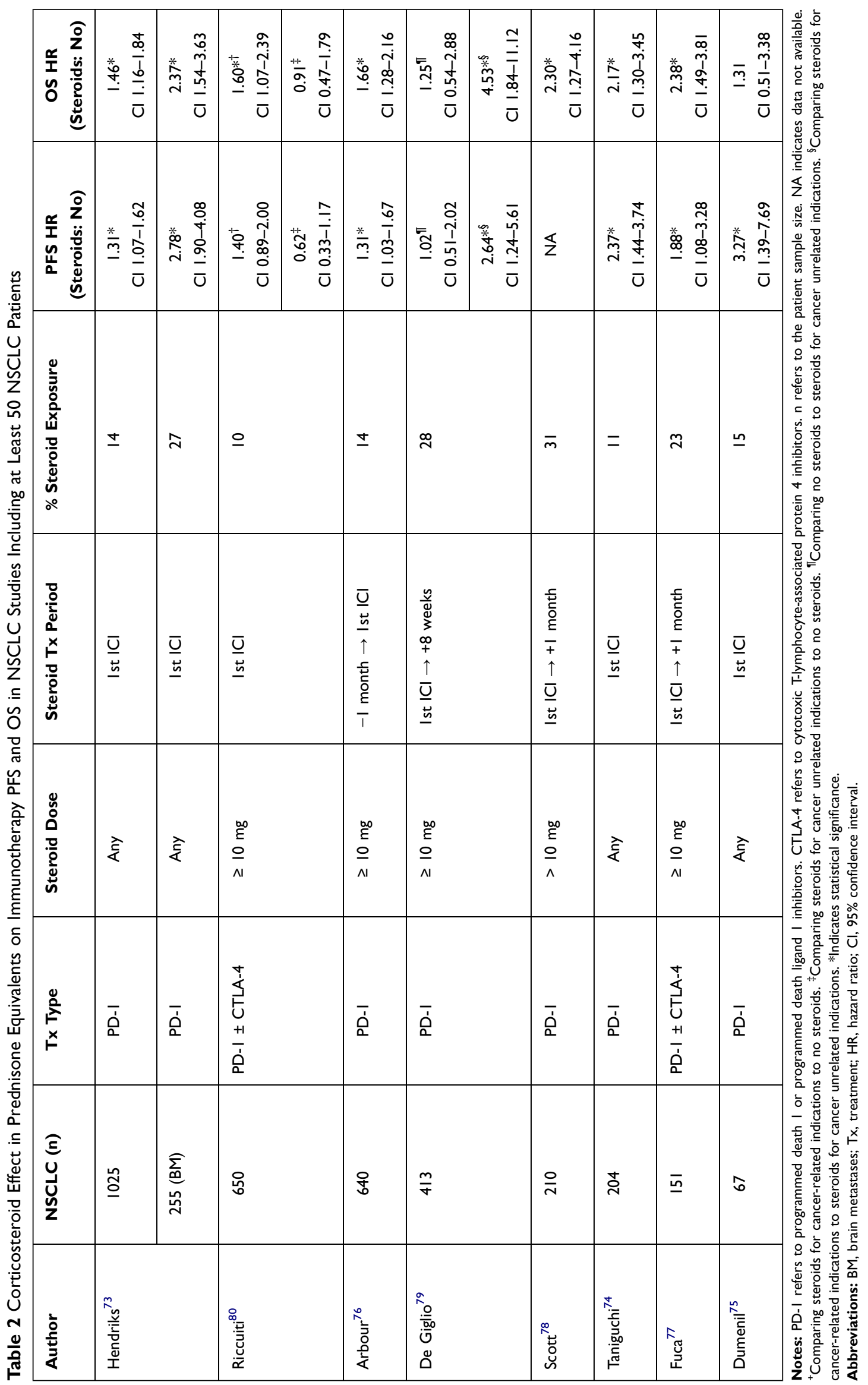


OS for those who developed an irAE while on ICI therapy for NSCLC. Compared to a control group of patients who did not develop an irAE or patients who developed an irAE but did not require the equivalent of prednisone $10 \mathrm{mg}$ daily or greater daily, those who required systemic corticosteroids for an irAE did not perform worse. However, within the group of patients who developed irAEs, those who required prednisone $10 \mathrm{mg}$ or more daily experienced worse median OS. On multivariate analysis, there was no difference in survival between patients with a grade 1-2 irAE vs 3-4 irAE; however, irAEs involving rash, pruritis, arthritis, and endocrinological dysfunction showed improvement. ${ }^{84}$ Taken together these data suggest that patients who develop certain irAEs that are less likely to require high dose systemic steroids have the best outcomes and those with irAEs that require systemic steroids may perform at least as well as patients who do not develop irAEs.

\section{Conclusion}

Since the identification of PD-L1 IHC as a partially predictive biomarker for ICI response in NSCLC, the field exploring additional biomarkers has expanded, attempting to explain why some patients with high PD-L1 tumors do not respond and others with PD-L1 negative tumors experience durable responses. This review explored a number of potential tumoral and patient factors which may be associated with immunotherapy outcomes; however, most require further prospective validation. With improvements to these biomarkers and the development of others, there may come a time when combinations of various immunotherapies and chemotherapies will be chosen to optimally treat patients based on a biomarker array.

\section{Disclosure}

Dr Lyudmila Bazhenova reports personal fees from Boehringer-Ingelheim. The authors report no other conflicts of interest in this work.

\section{References}

1. Global Burden of Disease Cancer, C, et al. Global, regional, and national cancer incidence, mortality, years of life lost, years lived with disability, and disability-adjusted life-years for 29 cancer groups, 1990 to 2017: a systematic analysis for the global burden of disease study. JAMA Oncol. 2019;5(12):1749-1768.

2. Reck M, Rodríguez-Abreu D, Robinson AG, et al. Pembrolizumab versus chemotherapy for PD-L1-positive non-small-cell lung cancer $N$ Engl J Med. 2016;375(19):1823-1833. doi:10.1056/NEJMoa1 606774
3. Gandhi L, Rodríguez-Abreu D, Gadgeel S, et al. Pembrolizumab plus chemotherapy in metastatic non-small-cell lung cancer. $N$ Engl $J$ Med. 2018;378(22):2078-2092. doi:10.1056/NEJMoa1801005

4. Hellmann MD, Paz-Ares L, Bernabe Caro R, et al. Nivolumab plus ipilimumab in advanced non-small-cell lung cancer. $N$ Engl $J$ Med. 2019;381(21):2020-2031. doi:10.1056/NEJMoa1910231

5. Reck M, Ciuleanu T-E, Dols MC, et al. Nivolumab (NIVO) + ipilimumab (IPI) +2 cycles of platinum-doublet chemotherapy (chemo) vs 4 cycles chemo as first-line (1L) treatment (tx) for stage IV/recurrent non-small cell lung cancer (NSCLC): checkmate 9LA. $J$ Clin Oncol. 2020;38(15):abstract. doi:10.1200/JCO.2020.38.1 5_suppl.9501

6. Tartarone A, Roviello G, Lerose R, et al. Anti-PD-1 versus anti-PDL1 therapy in patients with pretreated advanced non-small-cell lung cancer: a meta-analysis. Future Oncol. 2019;15(20):2423-2433. doi:10.2217/fon-2018-0868

7. Hirsch FR, McElhinny A, Stanforth D, et al. PD-L1 immunohistochemistry assays for lung cancer: results from Phase 1 of the Blueprint PD-L1 IHC assay comparison project. $J$ Thorac Oncol. 2017;12(2):208-222. doi:10.1016/j.jtho.2016.11.2228

8. Tsao MS, Kerr KM, Kockx M, et al. PD-L1 immunohistochemistry comparability study in real-life clinical samples: results of blueprint Phase 2 project. $J$ Thorac Oncol. 2018;13(9):1302-1311. doi:10.1016/j.jtho.2018.05.013

9. Paz-Ares L, et al. Outcomes with durvalumab by tumour PD-L1 expression in unresectable, stage III non-small-cell lung cancer in the PACIFIC trial. Ann Oncol. 2020;31(6):798-806. doi:10.1016/j. annonc.2020.03.287

10. Barlesi F, Vansteenkiste J, Spigel D, et al. Avelumab versus docetaxel in patients with platinum-treated advanced non-small-cell lung cancer (JAVELIN Lung 200): an open-label, randomised, Phase 3 study. Lancet Oncol. 2018;19(11):1468-1479. doi:10.1016/S1470-2045(18)30673-9

11. Borghaei H, Paz-Ares L, Horn L, et al. Nivolumab versus docetaxel in advanced nonsquamous non-small-cell lung cancer. $N$ Engl $J$ Med. 2015;373(17):1627-1639. doi:10.1056/NEJMoa1507643

12. Herbst RS, Baas P, Kim D-W, et al. Pembrolizumab versus docetaxel for previously treated, PD-L1-positive, advanced non-small-cell lung cancer (KEYNOTE-010): a randomised controlled trial. Lancet. 2016;387(10027):1540-1550. doi:10.1016/S0140-6736(15)01281-7

13. Rittmeyer A, et al. Atezolizumab versus docetaxel in patients with previously treated non-small-cell lung cancer (OAK): a phase 3, open-label, multicentre randomised controlled trial. Lancet. 2017;389(10066):255-265.

14. Brahmer J, Reckamp KL, Baas P, et al. Nivolumab versus docetaxel in advanced squamous-cell non-small-cell lung cancer. $N$ Engl J Med. 2015;373(2):123-135. doi:10.1056/NEJMoa1504627

15. Paz-Ares L, Luft A, Vicente D, et al. Pembrolizumab plus chemotherapy for squamous non-small-cell lung cancer. $N$ Engl $J$ Med. 2018;379(21):2040-2051. doi:10.1056/NEJMoa1810865

16. Mok TSK, Wu Y-L, Kudaba I, et al. Pembrolizumab versus chemotherapy for previously untreated, PD-L1-expressing, locally advanced or metastatic non-small-cell lung cancer (KEYNOTE-042): a randomised, open-label, controlled, phase 3 trial. Lancet. 2019;393(10183):1819-1830. doi:10.1016/S01406736(18)32409-7

17. Aguilar EJ, Ricciuti B, Gainor JF, et al. Outcomes to first-line pembrolizumab in patients with non-small-cell lung cancer and very high PD-L1 expression. Ann Oncol. 2019;30(10):1653-1659. doi:10.1093/annonc/mdz288

18. Fathi Z, Syn NL, Zhou J-G, et al. Molecular epidemiology of lung cancer in Iran: implications for drug development and cancer prevention. J Hum Genet. 2018;63(7):783-794. doi:10.1038/s10038018-0450-y

19. Rizvi NA, Hellmann MD, Snyder A, et al. Mutational landscape determines sensitivity to PD-1 blockade in non-small cell lung cancer. Science. 2015;348(6230):124-128. doi:10.1126/science.aaa1348 
20. Samstein RM, Lee C-H, Shoushtari AN, et al. Tumor mutational load predicts survival after immunotherapy across multiple cancer types. Nat Genet. 2019;51(2):202-206. doi:10.1038/s41588-018-0312-8

21. Hellmann MD, Ciuleanu T-E, Pluzanski A, et al. Nivolumab plus Ipilimumab in lung cancer with a high tumor mutational burden. $N$ Engl J Med. 2018;378(22):2093-2104. doi:10.1056/NEJMoa1801946

22. Paz-Ares L, Langer CJ, Novello S, et al. LBA80 Pembrolizumab (pembro) plus platinum-based chemotherapy (chemo) for metastatic NSCLC: tissue TMB (tTMB) and outcomes in KEYNOTE-021, 189, and 407. Ann Oncol. 2019;30:v917-v918. doi:10.1093/annonc/ mdz394.078

23. Herbst RS, Lopes G, Kowalski DM, et al. LBA79 Association between tissue TMB (tTMB) and clinical outcomes with pembrolizumab monotherapy (pembro) in PD-L1 positive advanced NSCLC in the KEYNOTE-010 and -042 trials. Ann Oncol. 2019;30:abstract. doi:10.1093/annonc/mdz394.077

24. Gandara DR, Paul SM, Kowanetz M, et al. Blood-based tumor mutational burden as a predictor of clinical benefit in non-small-cell lung cancer patients treated with atezolizumab. Nat Med. 2018;24 (9):1441-1448. doi:10.1038/s41591-018-0134-3

25. Peters S, et al. CTO74: tumor mutational burden (TMB) as a biomarker of survival in metastatic non-small cell lung cancer (mNSCLC): blood and tissue TMB analysis from MYSTIC, a Phase III study of first-line durvalumab \pm tremelimumab vs chemotherapy. AACR. 2019;79(13):abstract.

26. Al-Shibli KI, Donnem T, Al-Saad S, et al. Prognostic effect of epithelial and stromal lymphocyte infiltration in non-small cell lung cancer. Clin Cancer Res. 2008;14(16):5220-5227. doi:10.1158/10780432.CCR-08-0133

27. Brambilla E, Le Teuff G, Marguet S, et al. Prognostic effect of tumor lymphocytic infiltration in resectable non-small-cell lung cancer. J Clin Oncol. 2016;34(11):1223-1230. doi:10.1200/JCO.2015.63.0970

28. Zeng D-Q, Yu Y-F, Ou Q-Y, et al. Prognostic and predictive value of tumor-infiltrating lymphocytes for clinical therapeutic research in patients with non-small cell lung cancer. Oncotarget. 2016;7 (12):13765-13781. doi:10.18632/oncotarget.7282

29. Iglesia MD, et al. Genomic analysis of immune cell infiltrates across 11 tumor types. J Natl Cancer Inst. 2016;108(11).

30. Uryvaev A, Passhak M, Hershkovits D, et al. The role of tumor-infiltrating lymphocytes (TILs) as a predictive biomarker of response to anti-PD1 therapy in patients with metastatic non-small cell lung cancer or metastatic melanoma. Med Oncol. 2018;35(3):25. doi:10.1007/s12032-018-1080-0

31. Mazzaschi G, Madeddu D, Falco A, et al. Low PD-1 Expression in Cytotoxic $\mathrm{CD} 8+$ tumor-infiltrating lymphocytes confers an immune-privileged tissue microenvironment in NSCLC with a prognostic and predictive value. Clin Cancer Res. 2018;24 (2):407-419. doi:10.1158/1078-0432.CCR-17-2156

32. Vanderwalde A, Spetzler D, Xiao N, et al. Microsatellite instability status determined by next-generation sequencing and compared with PD-L1 and tumor mutational burden in 11,348 patients. Cancer Med. 2018;7(3):746-756. doi:10.1002/cam4.1372

33. Le DT, Uram JN, Wang H, et al. PD-1 blockade in tumors with mismatch-repair deficiency. $N$ Engl J Med. 2015;372 (26):2509-2520. doi:10.1056/NEJMoa1500596

34. Le DT, Durham JN, Smith KN, et al. Mismatch repair deficiency predicts response of solid tumors to PD-1 blockade. Science. 2017;357(6349):409-413. doi:10.1126/science.aan6733

35. De Marchi P, Berardinelli GN, Cavagna R, et al. EP1.04-11 Frequency of Microsatellite Instability (MSI) in Brazilian TKI non-treatable Non-Small Cell Lung Cancer (NSCLC) patients. J Thor Oncol. 2019;14(10):abstract. doi:10.1016/j.jtho.2019.08.2133

36. Warth A, Körner S, Penzel R, et al. Microsatellite instability in pulmonary adenocarcinomas: a comprehensive study of 480 cases. Virchows Arch. 2016;468(3):313-319. doi:10.1007/s00428-0151892-7
37. Gainor JF, Shaw AT, Sequist LV, et al. EGFR mutations and ALK rearrangements are associated with low response rates to PD-1 pathway blockade in non-small cell lung cancer: a retrospective analysis. Clin Cancer Res. 2016;22(18):4585-4593. doi:10.1158/1078-0432. CCR-15-3101

38. Mazieres J, Drilon A, Lusque A, et al. Immune checkpoint inhibitors for patients with advanced lung cancer and oncogenic driver alterations: results from the IMMUNOTARGET registry. Ann Oncol. 2019;30(8):1321-1328. doi:10.1093/annonc/mdz167

39. Lisberg A, et al. A Phase II study of pembrolizumab in EGFR-Mutant, PD-L1+, tyrosine kinase inhibitor naive patients with advanced NSCLC. $J$ Thorac Oncol. 2018;13(8):1138-1145.

40. Lee CK, Man J, Lord S, et al. Checkpoint inhibitors in metastatic EGFR-mutated non-small cell lung cancer-a meta-analysis. J Thorac Oncol. 2017;12(2):403-407. doi:10.1016/j.jtho.2016.10.007

41. Skoulidis F, Goldberg ME, Greenawalt DM, et al. STK11/LKB1 mutations and PD-1 inhibitor resistance in KRAS-mutant lung adenocarcinoma. Cancer Discov. 2018;8(7):822-835. doi:10.1158/ 2159-8290.CD-18-0099

42. Arbour KC, Jordan E, Kim HR, et al. Effects of co-occurring genomic alterations on outcomes in patients with kras-mutant non-small cell lung cancer. Clin Cancer Res. 2018;24(2):334-340. doi:10.1158/ 1078-0432.CCR-17-1841

43. Papillon-Cavanagh S, et al. STK11 and KEAP1 mutations as prognostic biomarkers in an observational real-world lung adenocarcinoma cohort. ESMO Open. 2020;5(2).

44. Rizvi N, Cho BC, Reinmuth N, et al. OA04.07 mutations associated with sensitivity or resistance to Immunotherapy in $\mathrm{mNSCLC}$ : analysis from the MYSTIC trial. J Thor Oncol. 2019;14(10):abstract. doi:10.1016/j.jtho.2019.08.428

45. Okamura R, et al. ARID1A alterations function as a biomarker for longer progression-free survival after anti-PD-1/PD-L1 immunotherapy. J Immunother Cancer. 2020;8(1).

46. Sun D, Tian L, Zhu Y, et al. Subunits of ARID1 serve as novel biomarkers for the sensitivity to immune checkpoint inhibitors and prognosis of advanced non-small cell lung cancer. Mol Med. 2020;26 (1):78. doi:10.1186/s10020-020-00208-9

47. Wang Z, Zhao J, Wang G, et al. Commutations in DNA damage response pathways serve as potential biomarkers for immune checkpoint blockade. Cancer Res. 2018;78(22):6486-6496. doi:10.1158/ 0008-5472.CAN-18-1814

48. Zhang J, et al. ZFHX3 mutation as a protective biomarker for immune checkpoint blockade in non-small cell lung cancer. Cancer Immunol Immunother. 2020;70:137-151.

49. Zhang $\mathrm{K}$, Hong $\mathrm{X}$, Song $\mathrm{Z}$, et al. Identification of deleterious $\mathrm{NOTCH}$ mutation as novel predictor to efficacious immunotherapy in NSCLC. Clin Cancer Res. 2020;26(14):3649-3661. doi:10.1158/ 1078-0432.CCR-19-3976

50. Gu X-B, Tian T, Tian X-J, et al. Prognostic significance of neutrophil-to-lymphocyte ratio in non-small cell lung cancer: a meta-analysis. Sci Rep. 2015;5(1):12493. doi:10.1038/srep12493

51. Jiang T, Bai Y, Zhou F, et al. Clinical value of neutrophil-tolymphocyte ratio in patients with non-small-cell lung cancer treated with PD-1/PD-L1 inhibitors. Lung Cancer. 2019;130:76-83. doi:10.1016/j.lungcan.2019.02.009

52. Hasegawa T, Yanagitani N, Utsumi H, et al. Association of High neutrophil-to-lymphocyte ratio with poor outcomes of pembrolizumab therapy in high-PD-L1-expressing non-small cell lung cancer. Anticancer Res. 2019;39(12):6851-6857. doi:10.21873/ anticanres. 13902

53. Li B, Huang X, Fu L. Impact of smoking on efficacy of PD-1/PD-L1 inhibitors in non-small cell lung cancer patients: a meta-analysis. Onco Targets Ther. 2018;11:3691-3696. doi:10.2147/OTT.S156421

54. Nishio M, et al. Multicentre phase II study of nivolumab in Japanese patients with advanced or recurrent non-squamous non-small cell lung cancer. ESMO Open. 2016;1(4):e000108. 
55. Kim JH, Kim HS, Kim BJ. Prognostic value of smoking status in non-small-cell lung cancer patients treated with immune checkpoint inhibitors: a meta-analysis. Oncotarget. 2017;8(54):93149-93155. doi:10.18632/oncotarget.18703

56. Mo J, Hu X, Gu L, et al. Smokers or non-smokers: who benefits more from immune checkpoint inhibitors in treatment of malignancies? An up-to-date meta-analysis. World J Surg Oncol. 2020;18(1):15. doi:10.1186/s12957-020-1792-4

57. Chiu M, Lipka MB, Bhateja P, et al. A detailed smoking history and determination of MYC status predict response to checkpoint inhibitors in advanced non-small cell lung cancer. Transl Lung Cancer Res. 2020;9(1):55-60. doi:10.21037/tlcr.2020.01.03

58. Chae YK, Davis AA, Raparia K, et al. Association of tumor mutational burden with DNA repair mutations and response to Anti-PD-1/ PD-L1 therapy in non-small-cell lung cancer. Clin Lung Cancer. 2019;20(2):88-96 e6. doi:10.1016/j.cllc.2018.09.008

59. Norum J, Nieder C. Tobacco smoking and cessation and PD-L1 inhibitors in non-small cell lung cancer (NSCLC): a review of the literature. ESMO Open. 2018;3(6):e000406. doi:10.1136/esmoopen2018-000406

60. Petrelli F, et al. Survival of patients treated with antibiotics and immunotherapy for cancer: a systematic review and meta-analysis. $J$ Clin Med. 2020;9(5).

61. Kaderbhai C, Richard C, Fumet JD, et al. Antibiotic use does not appear to influence response to nivolumab. Anticancer Res. 2017;37 (6):3195-3200. doi:10.21873/anticanres.11680

62. Hakozaki T, Okuma Y, Omori M, et al. Impact of prior antibiotic use on the efficacy of nivolumab for non-small cell lung cancer. Oncol Lett. 2019;17(3):2946-2952. doi:10.3892/ol.2019.9899

63. Huemer F, et al. Baseline absolute lymphocyte count and ECOG performance score are associated with survival in advanced non-small cell lung cancer undergoing PD-1/PD-L1 blockade. J Clin Med. 2019;8(7).

64. Huemer F, Rinnerthaler G, Westphal T, et al. Impact of antibiotic treatment on immune-checkpoint blockade efficacy in advanced non-squamous non-small cell lung cancer. Oncotarget. 2018;9 (23):16512-16520. doi:10.18632/oncotarget.24751

65. Zhao S, Gao G, Li W, et al. Antibiotics are associated with attenuated efficacy of anti-PD-1/PD-L1 therapies in Chinese patients with advanced non-small cell lung cancer. Lung Cancer. 2019;130:10-17. doi:10.1016/j.lungcan.2019.01.017

66. Derosa L, Hellmann MD, Spaziano M, et al. Negative association of antibiotics on clinical activity of immune checkpoint inhibitors in patients with advanced renal cell and non-small-cell lung cancer. Ann Oncol. 2018;29(6):1437-1444. doi:10.1093/annonc/mdy103

67. Pinato DJ, Howlett S, Ottaviani D, et al. Association of prior antibiotic treatment with survival and response to immune checkpoint inhibitor therapy in patients with cancer. JAMA Oncol. 2019;5 (12):1774-1778. doi:10.1001/jamaoncol.2019.2785

68. Galli G, Triulzi T, Proto C, et al. Association between antibiotic-immunotherapy exposure ratio and outcome in metastatic non small cell lung cancer. Lung Cancer. 2019;132:72-78. doi:10.1016/j.lungcan.2019.04.008

69. Tinsley N, Zhou C, Tan G, et al. Cumulative antibiotic use significantly decreases efficacy of checkpoint inhibitors in patients with advanced cancer. The Oncologist. 2020;25(1):55-63. doi:10.1634/ theoncologist.2019-0160

70. Imhann F, Bonder MJ, Vich Vila A, et al. Proton pump inhibitors affect the gut microbiome. Gut. 2016;65(5):740-748. doi:10.1136/ gutjnl-2015-310376

71. Routy B, Le Chatelier E, Derosa L, et al. Gut microbiome influences efficacy of PD-1-based immunotherapy against epithelial tumors. Science. 2018;359(6371):91-97. doi:10.1126/science. aan 3706
72. Jin Y, Dong H, Xia L, et al. The diversity of gut microbiome is associated with favorable responses to anti-programmed death 1 immunotherapy in Chinese patients with NSCLC. J Thorac Oncol. 2019;14(8):1378-1389. doi:10.1016/j.jtho.2019.04.007

73. Ouaknine Krief J, Helly de Tauriers P, Dumenil C, et al. Role of antibiotic use, plasma citrulline and blood microbiome in advanced non-small cell lung cancer patients treated with nivolumab. J Immunother Cancer. 2019;7(1):176. doi:10.1186/s40425-019-0658-1

74. Stevenson A, Panzica A, Holt A, et al. Host-microbe interactions mediating antitumorigenic effects of MRX0518, a gut microbiota-derived bacterial strain, in breast, renal and lung carcinoma. J Clin Oncol. 2018;36(15_suppl):abstract. doi:10.1200/ JCO.2018.36.15_suppl.e15006

75. Petrelli F, Bukovec R, Perego G, et al. Association of steroid use with survival in solid tumours. Eur J Cancer. 2020;141:105-114. doi:10.1016/j.ejca.2020.09.020

76. Hendriks LEL, Henon C, Auclin E, et al. Outcome of patients with non-small cell lung cancer and brain metastases treated with checkpoint inhibitors. $J$ Thorac Oncol. 2019;14(7):1244-1254. doi:10.1016/j.jtho.2019.02.009

77. Taniguchi Y, Tamiya A, Isa S-I, et al. Predictive factors for poor progression-free survival in patients with non-small cell lung cancer treated with nivolumab. Anticancer Res. 2017;37(10):5857-5862. doi:10.21873/anticanres. 12030

78. Dumenil $\mathrm{C}$, et al. Clinical factors associated with early progression and grade 3-4 toxicity in patients with advanced non-small-cell lung cancers treated with nivolumab. PLoS One. 2018;13(4):e0195945. doi:10.1371/journal.pone.0195945

79. Arbour KC, Mezquita L, Long N, et al. Impact of baseline steroids on efficacy of programmed cell death-1 and programmed death-ligand 1 blockade in patients with non-small-cell lung cancer. J Clin Oncol. 2018;36(28):2872-2878. doi:10.1200/JCO.2018.79.0006

80. Fuca G, Galli G, Poggi M, et al. Modulation of peripheral blood immune cells by early use of steroids and its association with clinical outcomes in patients with metastatic non-small cell lung cancer treated with immune checkpoint inhibitors. ESMO Open. 2019;4(1): e000457. doi:10.1136/esmoopen-2018-000457

81. Scott SC, Pennell NA. Early use of systemic corticosteroids in patients with advanced NSCLC treated with nivolumab. J Thorac Oncol. 2018;13(11):1771-1775. doi:10.1016/j.jtho.2018.06.004

82. De Giglio A, et al. Impact of Intercurrent Introduction of Steroids on Clinical Outcomes in Advanced Non-Small-Cell Lung Cancer (NSCLC) Patients under Immune-Checkpoint Inhibitors (ICI). Cancers (Basel). 2020;12(10).

83. Ricciuti $\mathrm{B}$, et al. Immune checkpoint inhibitor outcomes for patients with non-small-cell lung cancer receiving baseline corticosteroids for palliative versus nonpalliative indications. J Clin Oncol. 2019;37 (22):1927-1934.

84. Riudavets M, Mosquera J, Garcia-Campelo R, et al. Immune-related adverse events and corticosteroid use for cancer-related symptoms are associated with efficacy in patients with non-small cell lung cancer receiving Anti-PD-(L)1 blockade agents. Front Oncol. 2020;10:1677. doi:10.3389/fonc.2020.01677

85. Haratani K, Hayashi H, Chiba Y, et al. Association of immune-related adverse events with nivolumab efficacy in non-small-cell lung cancer. JAMA Oncol. 2018;4(3):374-378. doi:10.1001/jamaoncol.2017.2925

86. Grangeon $M$, et al. Association between immune-related adverse events and efficacy of immune checkpoint inhibitors in non-smallcell lung cancer. Clin Lung Cancer. 2019;20(10):201-207. doi:10.1016/j.cllc.2018.10.002

87. Ricciuti B, et al. Impact of immune-related adverse events on survival in patients with advanced non-small cell lung cancer treated with nivolumab: long-term outcomes from a multi-institutional analysis. J Cancer Res Clin Oncol. 2019;145(2):479-485. doi:10.1007/ s00432-018-2805-3 


\section{Publish your work in this journal}

Lung Cancer: Targets and Therapy is an international, peerreviewed, open access journal focusing on lung cancer research, identification of therapeutic targets and the optimal use of preventative and integrated treatment interventions to achieve improved outcomes, enhanced survival and quality of life for the cancer patient. Specific topics covered in the journal include: Epidemiology,

Submit your manuscript here: http://www.dovepress.com/lung-cancer-targets-therapy-journal detection and screening; Cellular research and biomarkers; Identification of biotargets and agents with novel mechanisms of action; Optimal clinical use of existing anticancer agents, including combination therapies; Radiation and surgery; Palliative care; Patient adherence, quality of life, satisfaction; Health economic evaluations. 